\section{L'Actualité économique}

L'ACTUALITÉ

ÉCONOMIQUE

\section{Filières de production : revue de la littérature et comparaison avec la théorie néo-classique}

Kh. Sekkat

Volume 63, numéro 1, mars 1987

URI : https://id.erudit.org/iderudit/601403ar

DOI : https://doi.org/10.7202/601403ar

Aller au sommaire du numéro

Éditeur(s)

HEC Montréal

ISSN

0001-771X (imprimé)

1710-3991 (numérique)

Découvrir la revue

Citer cet article

Sekkat, K. (1987). Filières de production : revue de la littérature et comparaison avec la théorie néo-classique. L'Actualité économique, 63(1), 118-142.

https://doi.org/10.7202/601403ar d'utilisation que vous pouvez consulter en ligne. 


\title{
Filières de production : revue de la littérature et comparaison avec la théorie néo-classique
}

\author{
Kh. SEKKAT \\ Département d'économie appliquée \\ de l'Université Libre de Bruxelles
}

INTRODUCTION

La notion de filière de production connaît depuis le début des années 1970 un grand succès (Rapport du Bureau d'information et de prévision économique (BIPE), 1977; numéro spécial des Annales des Mines, 1980; ...). Pourtant cette notion n'est pas nouvelle. En effet si on définit (provisoirement) la filière comme une succession d'étapes de production et de commercialisation, on constate que sa perception intuitive remonte à bien avant cette date ${ }^{1}$. Mais c'est l'École française d'économie industrielle qui en a fait un sujet d'analyse autonome. Le concept de filière est devenu un cadre de référence privilégié pour l'élaboration d'actions stratégiques et la description des enchaînements d'activités de production. Sorti graduellement du strict domaine académique pour atteindre les centres de décisions publics et privés, il bouscule les cadres habituels d'analyse à plusieurs égards :

- Étant conçu dans un environnement de concurrence imparfaite, il s'oppose à la vision walrasienne (Stoffaes, 1980) d'une économie où les entreprises ne peuvent, individuellement, influencer les prix et où l'allocation optimale des ressources est assurée par l'équilibre des marchés.

- Il oppose aux stratégies industrielles partielles classiques (Morvan, 1985) une vision globale qui prend en compte simultanément leurs différentes caractéristiques. Du point de vue de l'entreprise, la stratégie de filière englobe la vision en terme de marché (école du management) et celle en terme de technologie (école technique) (Creton, 1985). Au niveau du tissu industriel, le concept de filière offre une vision structurée et permet de renforcer la cohérence de l'ensemble en mettant en oeuvre l'ensemble des effets de synergie technologiques et commerciaux (Lorenzi-Truel, 1981).

Nous remercions H. Capron, M. Dewatripont, P. Kestens, J. Mercenier et F. Thys-Clément pour leurs commentaires et suggestions. Nous demeurons cependant seul responsable des erreurs. Cette étude a été réalisée grâce à un financement du Service de la Programmation Scientifique, Convention ARC 84/89-65.

1. Les premiers écrits sur l'inégration verticale par exemple datent du début du siècle. Et d'après Stoffaes (1980) le raisonnement en filière aurait guidé l'action des pouvoirs publics déjà au 18 ème siècle. 
- Au niveau descriptif, il déborde de la nomenclature du système productif conçue en branches et secteurs et transcende son découpage en primaire, secondaire et tertiaire.

Cependant si l'approche par filières est prometteuse, il faut être conscient de ses limites et de son champ d'utilisation :

- La multiplicité des définitions et des utilisations en ont fait un concept difficile à cerner et nous amène à noter qu'il n'existe pas une filière pertinente mais plusieurs (Jacquemin-Rainelli, 1984). La filière pertinente est différente selon l'agent économique qui s'y intéresse et les utilisations qu'il veut en faire. Elle ne trouve par conséquent tout son sens qu'en fonction de ces utilisations.

- Ensuite il serait erroné de croire que la filière n'est régie que par une dynamique et une logique bien propre et strictement indépendante de son environnement. La filière subit de l'influence et l'exerce sur cet environnement. Elle n'a donc toute sa signification que replacée dans un cadre plus vaste. De ce point de vue elle se situe incontestablement à la croisée des chemins de ceux qui privilégient la perspective d'une adaptation naturelle de l'industrie à l'environnement et ceux qui privilégient l'aspect stratégique de la manipulation de l'environnement.

Nous nous proposons dans ce travail d'effectuer une revue critique des principales contributions concernant le concept de filière. La réalisation d'une telle revue se heurte à deux catégories de problème :

- La théorie des filières est née et s'est développée presque exclusivement parmi les économistes francophones et demeure pratiquement inconnue du côté anglo-saxon. En outre aucune tentative d'intégration des approches néoclassiques formalistes dans l'analyse en filière n'a encore été entreprise. Loin d'être des substituts, ces deux théories sont à notre avis complémentaires et peuvent s'enrichir mutuellement. Un formalisme plus poussé est nécessaire pour éprouver la pertinence des résultats et arguments émanant de la théorie des filières ${ }^{2}$. L'intégration des concepts de la théorie néo-classique (contrôle vertical, coûts de transaction, contrats) permettra quant à elle de mieux préciser et clarifier ceux d'actions stratégiques dans les filières. Celles-ci enrichiront par contre le cadre néo-classique par tout l'aspect dynamique de la dominance et de la stratégie qu'il néglige très souvent.

- La plupart des auteurs qui se sont intéressés à ce sujet l'ont fait de façon ponctuelle sans que l'un ou l'autre ait suivi de près son développement depuis le début jusqu'à nos jours. En outre les écrits épars qui s'étalent sur plus d'une décennie traitent le plus souvent d'un des aspects en négligeant les autres $^{3}$. Par conséquent aucune réflexion globale sur le sujet n'a encore eu lieu pour permettre de jeter les bases d'une théorisation générale du concept.

2. La formalisation structurale de la filière entreprise par Lesage (1985) s'inscrit dans une optique purement descriptive et n'est pas (dans son état actuel) utilisable à cette fin.

3. La seule tentative de synthèse des diverses approches en filières est celle de Morvan (1985). 
L'objectif de ce travail est double : d'une part, offrir au lecteur une vue globale du concept et de ses multiples facettes et d'autre part, effectuer une plus grande intégration des théories néo-classiques et des filières. Nous nous efforcerons, en présentant les différentes approches et les liens qui existent entre elles, de montrer le niveau où chaque hypothèse est cruciale et les résultats qui y sont liés. Les échanges enrichissants avec la théorie néo-classique seront développés. Les critiques à l'égard des différentes approches seront mentionnées ainsi que les directions de recherches qui paraissent prometteuses.

Le texte sera organisé en six sections. La succession de celles-ci reflète la démarche d'un décideur (privé ou public) qui veut adopter une stratégie en filière. La première section a pour objet d'analyser les différentes définitions et d'en tirer les éléments constitutifs essentiels. Les approches pour l'identification des filières (sur lesquelles on va agir) sont exposées en deuxième section. La hiérarchisation de celles-ci selon des rapports de dominance-dépendance est discutée ensuite en section trois et les stratégies des acteurs (privés ou publics) sont analysées dans la quatrième section. Comme la rationalité de l'acteur suppose que pour fixer sa stratégie, il doit anticiper certains éléments tels que la réaction des autres ou l'évolution naturelle de la filière, la cinquième section se consacrera à la prévision dans les filières. Le travail se termine par une conclusion qui tente de dégager les axes de recherches futurs.

\section{DÉFINITIONS DU CONCEPT DE FILIĖRE}

Les définitions données de la filière sont multiples. Elles évoquent toutes une succession d'opérations de passage obligé qui s'emboîtent les unes dans les autres. Toutefois son appréhension diffère encore souvent d'auteur à auteur selon l'utilisation qu'ils veulent en faire. Au lieu de citer toutes les définitions existantes $^{4}$ nous nous contenterons, à l'instar de Lesage (1985) et Morvan (1985), de dégager les éléments constitutifs d'une filière et ses traits essentiels. Elle se définit par :

1) une succession d'opérations (ou d'ensembles d'opérations appelées plus généralement segments) distinctes, de passage obligé et orientée vers l'utilisation d'une ressource ou d'un produit donné ou la satisfaction d'une demande ;

2) à cette succession d'opérations est sous-jacente une suite d'actions d'acteurs correspondant à des segments. Les rapports entre les acteurs sont régis par un ensemble de relations techniques, financières, commerciales et sociales.

Commentons ces caractéristiques :

1) Il faut d'abord une succession d'opérations ou de segments et donc un ordre dans le passage ou l'évolution à travers les différentes étapes. Ils sont distincts même si leurs frontières ne sont pas définies de la même façon par

4. Un inventaire de ces définitions peut être trouvé dans la publication de l'Association pour le développement des études sur la firme et l'industrie, ADEFI (1985) et dans Monfort (1983). 
les différents auteurs. Pour le BIPE, par exemple, la frontière entre deux segments est la séparation par un marché. Mais alors en cas d'intégration verticale complète de la filière (comme c'est le cas pour certaines compagnies pétrolières), celle-ci se réduira à un seul segment. D'autres identifient un segment à une opération (technique ou commerciale) finement définie. Mais cette finesse poussée à son extrême conduira à un nombre important de segments sans améliorer nécessairement l'analyse. Nous considérons pour notre part qu'il n'est pas nécessaire de donner une définition préalable et universelle d'un segment. La définition dépendra du genre d'analyse que l'on veut mener. Il n'est en effet pas intéressant pour un macroéconomiste, qui s'intéresse aux grands agrégats, de décomposer sa filière en stades techniques très fins allant jusqu'à distinguer les différentes opérations au sein d'une section de production. Par contre une décomposition sur base de branches et de secteurs ne sera pas de beaucoup d'utilité pour l'ingénieur ou le gestionnaire qui voudrait étudier son environnement immédiat pour déterminer sa stratégie de production ou de marché. La filière contiendra par conséquent plus ou moins de segments selon le besoin.

Le passage par ces segments est obligé. Mais la notion de passage obligé ne signifie pas une «rigidité » dans le processus de production. Elle implique uniquement une logique dans l'organisation de la filière où les segments s'imbriquent les uns dans les autres pour former un « tout » cohérent. Il peut évidemment exister plus d'un chemin qui conduit au même résultat. Ces chemins peuvent être multiples pour deux raisons : à cause du niveau d'agrégation ou à cause des possibilités de substitution des inputs. Dans le premier cas on pourrait réduire le nombre de chemins possibles en affinant la définition de l'objectif et des segments. On n'arrive cependant pas nécessairement à un chemin unique du fait de la substituabilité des inputs. Les filières seront par conséquent :

- plus ou moins épaisses (Morvan, 1985) selon le niveau d'agrégation et les possibilités de substitution ;

- arborescentes plutôt que linéaires à cause surtout de la substituabilité ;

- potentiellement interdépendantes car des segments peuvent irriguer plusieurs filières.

La filière est orientée. Le passage obligé par des segments distincts a un but : l'utilisation d'une matière ou la satisfaction d'une demande finale. Dès lors on rencontre couramment deux types de filières dans la littérature : la filière produit et la filière demande finale.

a) Le critère d'appartenance au premier type de filières repose sur une caractéristique physique, chimique ou technique de production (filière bois, filière phosphate, ...). L'identification de la filière produit part d'un produit bien déterminé (bois, fer, charbon, ...) et suit son passage par différentes étapes jusqu'aux différentes utilisations finales qui en sont faites (de l'amont vers 
l'aval). Elle correspond plus à une vision technique du concept. Elle s'avère dès lors (Tollet, 1982) peu opérationnelle pour expliquer et prévoir les transformations du système productif ${ }^{5}$.

b) L'appartenance au deuxième type de filières (la filière demande finale) repose sur le concours des différents segments à la satisfaction d'une demande finale (filière habillement, filière information, filière équipement, filière transport, ...). Partant d'une fonction de demande finale (consommation, investissement, exportation) définie dans une nomenclature assez fine, on identifie la filière en dégageant les segments qui concourent à sa satisfaction depuis l'aval (très près du demandeur final) jusqu'à l'amont (matière première). La filière demande finale, définie ainsi, correspond donc mieux aux processus de décision et de prévision dans l'entreprise que la filière produit. Alors que la filière produit n'est qu'une modalité de découpage du système productif, la filière demande finale apparaît, en outre, comme une méthode d'analyse de la politique d'acteurs (publics ou privés). Nous nous intéresserons par conséquent dans la suite de ce travail exclusivement à la filière demande finale.

2) À chaque stade ou opération correspond un acteur qui par son action et/ou sa réaction anime la filière et la « dynamise ». Cette caractéristique replace la filière dans son contexte actif et en fait autre chose qu'une mécanique. Elle permet par conséquent de considérer des stratégies qui animent, font et défont des filières. Ces différents acteurs sont liés non seulement par des relations techniques et commerciales mais aussi par des rapports sociaux et financiers qui permettent de mieux appréhender l'évolution du système dans son ensemble.

\section{DÉTERMINATION EMPIRIQUE DES FILIÈRES}

Avant de se livrer à des analyses stratégiques ou à des prévisions sur la filière, l'acteur doit la connaître. La détermination des filières apparaît donc comme un préalable à toute ébauche d'analyse stratégique ou prévisionnelle (Jacquemin-Rainelli, 1984). Elle consiste à structurer les branches d'activité d'une économie donnée en dégageant les principales relations verticales. Nous allons présenter dans ce qui suit les approches et méthodes pour la détermination empirique des filières avant de mentionner les critiques s'y rapportant.

1. Approches de détermination empirique des filières

Pour le découpage du système productif en filières l'acteur dispose de deux approches :

- Approche monographique : se concentre sur la détermination et l'analyse d'une filière bien déterminée. Elle descend au niveau microéconomique pour suivre un ou des produits et leurs évolutions (Monfort-Dutailly, 1983). Elle cherche par conséquent à dégager plus des articulations techniques que

5. Ce point sera éclairci dans la section $\mathrm{V}$. 
commerciales. Elle répond surtout à des préoccupations de gestionnaire d'entreprise pour l'orientation de sa stratégie. L'application de cette méthode nécessite soit le concours d'ingénieurs spécialisés soit l'enquête auprès des entreprises.

- Approche statistique : c'est une approche plus macroéconomique. La plupart des travaux réalisés dans le cadre de cette approche procèdent à un redécoupage du tableau entrées-sorties. Ils ne portent pas sur un produit particulier mais cherchent à dégager pour l'économie entière quelques grands arbres essentiels autour desquels s'ordonnent la plupart des activités.

\section{Méthodes de l'approche statistique}

Les méthodes utilisables dans le cadre de cette approche sont nombreuses (Monfort-Dutailly, 1983). Nous nous contenterons de présenter celles proposées par Monfort (1983). Ce sont en effet les seules qui sont directement liées aux filières et qui ont donné lieu à des applications en France et en Belgique. Elles sont au nombre de quatre et s'appliquent toutes aux données des tableaux entréessorties $\left(X_{i j}\right)$.

\section{a) Agrégation par l'aval}

Soit $X_{i j}$ le montant absolu des achats de la branche $j$ en produit $i$ et $e_{i}$ les emplois totaux du produit $i$. La branche $i$ fait partie de la même filière que la branche $j$ (i est agrégée à $j$ ) si :

i) $j$ représente le principal débouché de $i$ :

$$
X_{i j}=\max _{k} X_{i k}
$$

ii) $j$ représente une part suffisante des débouchés de $i$ :

$$
\frac{X_{i j}}{e_{i}}>C
$$

où $C$ est un coefficient fixé a priori.

\section{b) Agrégation par l'amont}

Le principe de l'agrégation par l'amont est le même que celui de l'agrégation par l'aval, mais on se base sur les achats au lieu des ventes. 
c) Sensibilité aux variations absolues de la demande finale

Soit $T$ la matrice des coefficients techniques dont l'élément $t_{i j}$ est tel que $t_{i j}=\frac{X_{i j}}{P_{j}}$ où $P_{j}$ est la production de la branche $j$.

La matrice $A=(\mathrm{I}-T)^{-1}$ mesure l'impact sur chaque branche d'une variation de la demande finale.

L'idée de base qui inspire cette méthode est que les branches appartenant à une même filière devront réagir de façon semblable à une impulsion de la demande finale. La proximité des réactions est mesurée par :

$$
d(i, j)=\sum_{k}\left(a_{i k}-a_{j k}\right)^{2}
$$

où $a_{i j}$ sont les éléments de $A$.

Les branches $i$ et $j$ pour lesquelles cette distance est minimale sont agrégées (font partie de la même filière).

d) Sensibilité aux variations relatives de la demande finale

Le principe de cette méthode est le même que celui de la précédente sauf qu'on se base sur les variations relatives de la production par rapport aux variations relatives de la demande finale (élasticité de la production par rapport à la demande).

Pour chacune de ces quatre méthodes la procédure de détermination de la filière est itérative (Monfort-Dutailly, 1983).

\section{Critiques relatives aux méthodes statistiques}

Ces différentes méthodes statistiques relèvent des limites (Morvan, 1985 ; Tollet-Kestens, 1981) qu'on peut regrouper en deux catégories. La première a trait aux informations statistiques de base, alors que la deuxième porte sur les méthodes elles-mêmes. En effet :

i) Les informations statistiques restent encore souvent trop agrégées pour faire apparaître les liens plus spécifiques des différentes activités ${ }^{6}$. En outre les données statistiques ne sont généralement pas construites pour les besoins de détermination des filières. Enfin dans certains pays les tableaux entrées-sorties ne sont publiés qu'avec plusieurs années de retard (huit à neuf dans le cas belge).

ii) Les méthodes elles-mêmes ont été critiquées pour plusieurs raisons:

- Elles privilégient presque uniquement les rapports en termes d'achat vente puisqu'elles portent sur des données quantitatives de flux d'échanges, risquant ainsi de donner une vue réductrice des relations entre les agents

6. Ce problème d'agrégation n'est pas spécifique aux filières. 
en ne dégageant que des filières marchandes. Cette critique ne nous semble toutefois pas pertinente. Le découpage du système productif sur base de ces méthodes ne représente en effet qu'un aspect de l'analyse en filière, qui devra être complétée par des aspects stratégiques et d'analyse de la dominance.

- Les deux premières méthodes se basent sur des seuils d'acceptation arbitraires. Les filières obtenues sont alors toujours conditionnelles à ces seuils arbitraires.

- Les filières dégagées sur base de ces méthodes sont presque toujours linéaires et indépendantes alors que dans la réalité industrielle certaines se croisent et son arborescentes.

\section{LA DOMINANCE DANS LES FILIĖRES DE PRODUCTION}

La théorie des filières de production est conçue dans un environnement de concurrence imparfaite. L'imperfection des marchés confère à certains acteurs un pouvoir sur les autres (Soulié, 1980). De cette asymétrie des pouvoirs résulte que certains intervenants peuvent imposer à d'autres des conditions qui favorisent leur propre intérêt (de ceux qui ont le pouvoir). Ils sont alors qualifiés de dominants. L'étude et l'identification des segments dominants dans une filière présentent donc un intérêt certain pour l'acteur (privé ou public) qui établit sa stratégie et pour l'économiste qui analyse le comportement des différents intervenants. Il est par conséquent intéressant de pouvoir hiérarchiser une filière en fonction des rapports dominance-dépendance. Pour ce faire il faut répondre à quatre questions :

1. Comment définir la dominance?

2. Quelle est sa dynamique si elle en a une ?

3. Comment l'étudier empiriquement ?

4. Quelles sont ses sources?

Le BIPE ayant été très loin dans la recherche des réponses aux trois premières questions, nous nous baserons dès lors sur ses travaux pour y répondre. Par contre, la dernière question n'a reçu que peu d'attention du BIPE, plus préoccupé par les résultats de la dominance que par son origine. Nous ferons donc largement appel à la théorie néo-classique pour établir les sources de dominance.

\section{Définition de la dominance}

Les définitions de la dominance utilisées pour hiérarchiser une filière varient selon les auteurs (Aujac, 1960 ; BIPE, 1977 ; Gazon, 1976 ; Lantner, 1974) ${ }^{7}$. Cependant si la plupart de ces définitions permettent une hiérarchisation dans la transmission de l'influence économique on ne peut en inférer pour autant une structure de dominance. En effet celles-ci ne considèrent pas explicitement le

7. La plupart ne parlaient pas en termes de filière. 
problème des imperfections des marchés. C'est dans cette direction que se sont orientés les tavaux du BIPE (1977). Pour caractériser la dominance dans une filière, le BIPE pose deux hypothèses dont la première définit l'aspect statique et la deuxième l'aspect dynamique :

- Un acteur dans une filière (producteur ou distributeur) tire sa capacité d'action et de négociation du contrôle qu'il peut exercer sur le marché final. Le segment qui confère à son acteur cette aptitude est donc dit dominant. Le contrôle du marché final est déterminé par l'aptitude à orienter l'utilisateur final vers des exigences qui correspondent plus aux capacités techniques et économiques (coûts, technologies, performances, prix) de celui qui l'exerce.

- La localisation du pouvoir de domination (aptitude à contrôler) varie selon le degré de développement du marché final. Au fur et à mesure que celui-ci évolue depuis l'apparition du produit (phase de développement) ${ }^{8}$ jusqu'à sa maturité, l'aptitude de contrôle (et donc de dominance) se déplace de l'amont vers l'aval de la filière (en passant par le milieu).

Le pouvoir de domination est donc l'aptitude à contrôler et orienter l'utilisateur final selon les intérêts de celui qui l'exerce. Toutefois ce pouvoir peut aussi provenir du contrôle d'éléments sans lesquels l'ensemble ne pourra être réalisé (Morvan, 1985). Ainsi le monopoleur d'une matière première contrôlera son marché final en imposant son prix à tous les stades avals qui eux sont en concurrence et ceci sans jouer sur les caractéristiques techniques du produit final et indépendamment de la phase du cycle.

\section{Aspect dynamique de la dominance}

La localisation de la dominance n'est pas rigide mais se déplace dans le temps à travers les différents segments. Le BIPE présente la dynamique de la dominance parallèlement à l'évolution du cycle de vie du produit de la façon suivante :

- En phase de développement : le produit est nouveau, ses perspectives de vente croissantes, et le rôle de l'innovation technologique et des performances techniques est très important. Les ventes sont néanmoins encore modérées nécessitant un appareil de production flexible. C'est le segment amont, là où se fait généralement le plus de recherche-développement, qui maîtrise ces éléments (innovations, performances, ...). Les acheteurs du produit final, peu nombreux, généralement très informés et avec de fortes exigences techniques, connaissent le producteur amont qui utilise sa propre marque. Les acteurs des stades suivants (centre et aval) ne font alors que s'adapter aux évolutions techniques de l'amont qui domine. Ils sont en concurrence et leur survie dépend de leur aptitude à intégrer ces évolutions.

8. Cette typologie renvoie, comme on le verra par la suite, à la théorie du cycle de vie du produit. 
- En phase de diffusion : le produit tend vers une certaine banalisation technique, le marché final s'élargit (consommation de masse). Le pouvoir de domination se déplace vers le centre de la filière où se trouvent les segments intermédiaires qui, grâce à leur taille, peuvent satisfaire un marché aussi large. Le producteur qui a les moindres coûts détermine le prix. Une situation d'oligopole règne dans le centre de la filière alors que l'aval est en concurrence.

- En phase de maturité : le produit, maintenant tout à fait banalisé, est vendu sur un marché très large et en faible croissance. L'importance des segments de distribution devient très grande. Le pouvoir de domination se déplace vers l'aval qui est en contact direct avec le consommateur, maîtrise les techniques de promotion, la publicité et le service après vente. À ce stade le pouvoir de l'aval peut être tel qu'il impose sa propre marque au produit et rend les producteurs-amont tout à fait inconnus de l'utilisateur final ${ }^{9}$.

Cette typologie de l'évolution de la domination dans les filières représente la dynamique naturelle et rejoint, par là, le raisonnement des tenants d'une adaptation naturelle de l'industrie aux conditions de l'environnement. Cependant cette dynamique naturelle est théorique et risque d'être rompue notamment par diverses stratégies industrielles privées ou publiques (Jacquemin, 1985) ${ }^{10}$. Par conséquent la domination dans les filières se trouve, comme on l'a noté dans l'introduction, à la croisée des chemins de ceux qui privilégient l'adaptation naturelle et ceux qui ne considèrent que l'aspect stratégique ${ }^{11}$. La morphologie d'une filière à un moment donné sera dès lors déterminée par l'interaction entre ces deux tendances.

\section{Détection de la dominance}

À partir de l'observation de plusieurs filières françaises, le BIPE a dégagé une batterie d'indicateurs qui permettent de déterminer, pour une filière étudiée, les rapports de domination. Certains de ces indicateurs sont quantitatifs et d'autres, qualitatifs, et permettent donc d'appréhender mieux la complexité de la réalité industrielle. Ils sont ${ }^{12}$ au nombre de dix-sept et répartis en trois grandes catégories :

a) Les indicateurs de la phase du cycle de vie du produit (au nombre de quatre) révèlent, sur base de la deuxième hypothèse posée par le BIPE pour caractériser la dominance, dans laquelle des trois grandes parties de la filière (amont, centre, aval) devrait théoriquement se situer le pouvoir de domination (voir les aspects dynamiques de la dominance).

9. Remarquons pour être complet que cette application de la théorie du cycle de vie du produit qui consiste à lier technique de production et technique de marketing a eu beaucoup d'échos OutreAtlantique notamment avec les travaux de Hayes-Wheelwright (1979a, 1979b).

10. Voir la section IV.

11. Pour une discussion plus avancée de ces deux tendances, voir Jacquemin (1985).

12. Une présentation complète en est donnée dans BIPE (1977) et Tollet (1982). 
b) Les indicateurs de localisation du pouvoir de domination (au nombre de huit) permettent de mieux localiser le pouvoir de domination car dans la réalité, la typologie cycle de vie peut comporter des exceptions et une filière peut avoir plus de trois segments. Ces critères cherchent à évaluer le degré de concentration et de monopolisation-monopsonisation relatif sur les marchés. C'est en effet le développement de concurrence dans les différents stades de la filière avec l'évolution du cycle de vie du produit, qui conditionne la localisation de la dominance (Malsot, 1980).

c) Le dernier groupe de critères (au nombre de cinq) porte notamment sur les relations entre acteurs de deux segments successifs ainsi que leurs rapports avec le marché final. Ces indicateurs ont pour but de déterminer s'il y a exercice effectif du pouvoir de dominance entre les deux acteurs.

Dans la pratique l'utilisation de ces indicateurs pose différents problèmes :

- Plusieurs de ces indicateurs sont difficiles à évaluer (à moins de se livrer à des enquêtes approfondies) et on est souvent amené à se contenter seulement d'une partie.

- Des contradictions peuvent apparaître entre les indicateurs car :

i) La satisfaction d'une fonction de demande finale peut être réalisée par plusieurs produits substituables mais qui se situent chacun à un stade différent de leur cycle de vie. On propose dans ce cas d'utiliser une nomenclature de demandes finales plus fine pơur résoudre le problème. Mais si cette solution permet de surmonter ces difficultés dans certains cas, elle ne peut les éliminer complètement.

ii) Certains acteurs de la filière essaient d'exercer un pouvoir de domination même si l'évolution du cycle de vie ne le leur confère pas. L'intégration verticale est une des pratiques les plus couramment rencontrées à cet égard.

\section{Sources de la dominance et concurrence imparfaite}

La théorie des filières apporte à l'analyse un élément très important qui est l'organisation à tout moment d'une filière selon des rapports de dominancedépendance. Toutefois le concept de dominance lui-même reste assez mal expliqué dans la littérature et notamment en ce qui concerne ses origines. On s'est plus intéressé à étudier les conséquences de la dominance qu'à chercher les fondements théoriques de celle-ci. Le BIPE bien qu'ayant été loin dans l'étude de la dominance base sa méthodologie entièrement sur l'observation empirique et très peu sur des principes théoriques. Cette méthodologie est par conséquent inutilisable pour toute analyse ou formalisation théorique. Ce manque nous semble pourtant susceptible d'être comblé par une plus grande intégration entre la théorie néo-classique de la concurrence imparfaite et la théorie des filières. Afin de réaliser cette intégration deux questions se présentent à l'esprit : 
i) Si le pouvoir de domination provient du contrôle du marché final, quels sont les éléments d'imperfection de marché qui permettent ce contrôle ?

ii) Quel est l'apport de la dominance (présentée dans le cadre des filières) par rapport à la théorie néo-classique de la concurrence imparfaite ?

i) Les réponses à la première question sont nombreuses (BIPE, 1977 ; Morvan, 1985 ; Stoffaes, 1980) et portent principalement sur :

- La détention d'un brevet d'invention, constituant ainsi une barrière légale à l'entrée (Tirole, 1985) ;

- La possession d'un secret de fabrication (exemple : Coca-Cola) ;

- L'existence d'avantages technologiques spécifiques de certaines entreprises leur permettant de faire des profits sans générer l'entrée (Tirole, 1985) ;

- La situation de monopole naturel où le nombre d'utilisateurs et la technologie sont tels qu'il ne peut y avoir qu'une entreprise produisant de manière efficace à ce stade de production (à cause d'économie d'échelle par exemple) ;

- L'existence d'une clientèle importante avec laquelle s'établit un contact privilégié. L'effet clientèle est d'autant plus important que l'information de la clientèle est imparfaite et que les coûts associés au changement d'entreprises fournisseurs sont importants. Remarquons qu'en cas de soustraitance il peut exister un effet « fournisseur » analogue mais dans le sens inverse ;

- La localisation privilégiée sur un espace géographique ;

- La barrière à l'entrée générée par l'importance des investissements à réaliser avant de s'établir.

ii) Comme réponse à la deuxième question nous considérons que l'apport le plus important de la notion de domination dans les filières par rapport à la concurrence imparfaite ${ }^{13}$ est la dynamique de la dominance. Alors que dans cette dernière l'existence du pouvoir d'intervenants sur les autres est ad hoc, dans le cadre de l'analyse des filières il est, sous réserves d'acceptation des hypothèses, explicite et évolue suivant un schéma bien précis. Ce schéma, que nous avons appelé précédemment évolution naturelle, donnera lieu à des comportements stratégiques des acteurs soit pour s'y adapter soit pour le manipuler à leur avantage (voir la quatrième section). Ce dernier aspect ouvre par conséquent la porte à des exercices de prévision du comportement stratégique des acteurs comme on le verra dans les sections suivantes.

13. Du moins au niveau microéconomique. 


\section{L'ASPECT STRATÉGIQUE DU CONCEPT}

Nous avons vu que les segments d'une filière sont liés très étroitement par le flux d'échanges de biens du fait de leur concours à la satisfaction d'une même demande et par des rapports de dominance-dépendance dus à la différence de leur pouvoir de contrôle du marché final. Ces deux types de liens sont par conséquent à la base des comportements stratégiques des divers acteurs.

Les liens par les flux d'échanges (en l'absence de tout pouvoir de domination) feront que dans certains cas, les acteurs auront tous intérêt à adopter une stratégie de communication et de développement harmonieux de tous les segments (effet de synergie). Les liens par les rapports de domination-dépendance ainsi que leur évolution temporelle donneront lieu à des actions stratégiques des acteurs visant à se placer constamment sur les niveaux dominants.

Suite à ces considérations la stratégie de filière apparaît plus globale que des stratégies classiques comme la diversification, la concentration ou l'intégration verticale. La stratégie de concentration joue sur l'effet taille pour peser sur les coûts et le pouvoir de négociation. L'intégration verticale, au sens strict, privilégie des considérations physiques alors que la stratégie de diversification, qui cherche à limiter l'effet du risque, se base quant à elle sur des considérations purement financières. La stratégie de filière (pour un groupe par exemple) en revanche prend en compte une partie de toutes ces motivations. Elles sont d'ordre physique puisque la stratégie de filière considère que le développement harmonieux de l'ensemble des segments conduira à un résultat meilleur que la somme des résultats des différents segments (effet de synergie). Elles sont aussi d'ordre financier car en réorientant en permanence les capitaux entre les différents segments on pourrait assurer un bon fonctionnement de l'ensemble et une rentabilité maximale à long terme ${ }^{14}$.

Par ailleurs l'approche stratégique des filières est pertinente aussi bien au niveau de l'entreprise individuelle qu'au niveau national. La plupart des stratégies d'entreprise, qui n'altèrent pas le bien-être social, devront d'ailleurs être acceptées et même encouragées par les pouvoirs publics au niveau national (voir Rey-Tirole, 1986 ; Warren-Boulton, 1979 ; Williamson, 1975) car elles ont des effets qui rejoignent ceux recherchés par ceux-ci. Nous présenterons donc en premier lieu l'approche pour l'entreprise et analyserons ensuite les éléments nouveaux attachés au caractère national de la stratégie.

\section{Stratégie de l'entreprise}

Nous traiterons successivement la stratégie sans pouvoir de marché (lien par flux d'échanges uniquement), avec pouvoir de marché (avec asymétrie des pouvoirs) et dans une perspective dynamique.

14. Le cas japonais présenté ci-dessous illustre bien cette idée. 


\section{a) Stratégie sans pouvoir de marché}

Même dans un environnement concurrentiel où il n'y a pas d'asymétrie de pouvoir entre les acteurs, ceux-ci peuvent avoir intérêt à adopter une stratégie en terme de filière. Le développement cohérent de l'ensemble donnera en effet sous certaines conditions un meilleur résultat que la somme des résultats individuels. Les arguments théoriques qui justifient ces propos se trouvent dans les divers travaux sur le contrôle vertical. Bien que la plupart des études soient développées dans le cas d'intégration verticale complète, il existe presque toujours des substituts contractuels à celle-ci. Nous nous contenterons ici de présenter très brièvement ces arguments et renvoyons le lecteur aux articles cités pour de plus amples informations. Une grande coordination entre les segments successifs peut être profitable à tous car :

- Les interdépendances technologiques pour certains produits font qu'une bonne coordination dans le temps et l'espace de la production diminue les coûts. L'intégration verticale peut être un bon moyen pour réaliser cet objectif. Cependant comme le fait remarquer Richardson (1972) à chaque stade de production peuvent correspondre des compétences particulières. L'acteur qui s'intègre verticalement peut ne pas avoir ces compétences pour gérer le stade intégré. L'intégration risque alors d'être inefficace. Comme en outre l'élément technologique lui-même ne requiert pas le transfert de propriété, cette coordination peut être réalisée en passant toujours par un marché (Warren-Boulton, 1979).

- Les différents acteurs ne disposent pas toujours de la même information (information asymétrique). Des informations sur la demande ou les coûts (des autres stades de production), détenues par une partie des acteurs seulement et que les mécanismes du marché ne révèlent pas, peuvent être utiles à d'autres acteurs. Une meilleure communication de l'information entraînera une diminution des coûts et un ajustement plus rapide aux changements de la demande et des coûts. L'intégration verticale, en éliminant le désir d'utilisation stratégique de cette information, incite à la révéler et entraîne donc une plus grande efficacité de la production (Crocker, 1983). Si le gain réalisé grâce à une meilleure communication de l'information est suffisant, les mêmes résultats pourront être atteints par d'autres moyens contractuels que l'intégration verticale (Warren-Boulton, 1979).

- Il existe des coûts de transactions. Ce problème a donné lieu à un traitement très détaillé par Williamson (1975). Les coûts de transactions sont dus aux problèmes d'information exposés ci-dessus et à la négociation et la recherche de clients et fournisseurs. Ces coûts peuvent être importants à cause d'éléments d'environnement (incertitude, complexité) et d'éléments humains (rationalité limitée, opportunisme). L'intégration verticale peut diminuer ces coûts. Mais le même résultat peut être atteint par des contrats d'approvisionnement et des concessions exclusives. 
- L'incertitude peut avoir un impact sur les performances globales des différents stades et sur leurs coûts. Différents modèles traitent de ces problèmes (Arrow, 1975 ; Bernhardt, 1977 ; Carlton, 1979). Le modèle proposé par Arrow (1975) traite de l'impact d'incertitude sur les conditions d'offre et son rôle comme incitant à l'intégration verticale qui permet de réaliser une plus grande efficacité de la production. L'intégration n'est cependant pas nécessaire car peut être remplacée par des moyens contractuels si on lève certaines de ses hypothèses (Blair-Kaserman, 1983). L'incertitude sur la demande a été traitée par Bernhardt (1977) et Carlton (1979). Les fluctuations de la demande imposent (Bernhardt, 1977) des coûts par la nécessité de détenir des stocks et d'adopter une technologie de production flexible. Ces coûts peuvent être réduits par des moyens contractuels entre les segments. Avec un modèle différent de Bernhardt, Carlton (1979) montre quant à lui que les firmes trouveront profitable de contrôler leurs inputs par intégration ou contrat s'il y a incertitude sur la demande. Les modèles présentés ci-dessus laissent cependant de côté le problème important de la différence du degré d'aversion au risque entre les acteurs. Une analyse détaillée de ce problème est présentée par Blair-Kaserman (1983). Ils montrent comment la différence du degré d'aversion au risque entre les acteurs de deux stades de production successifs conduit à des inefficacités dans la production. Ils montrent que ces inefficacités peuvent être réduites par contrôle vertical (intégration ou contrat).

\section{b) Stratégie avec pouvoir de marché}

La stratégie de filière en situation non concurrentielle repose sur l'existence d'un pouvoir de domination (première hypothèse pour caractériser la dominance). De cette hypothèse on peut dégager deux types de comportements stratégiques selon que l'acteur a ou n'a pas le choix et les moyens pour sa localisation dans la filière :

i) Si l'acteur a le choix et les moyens pour sa localisation dans la filière sa stratégie consistera, non à intégrer tous les segments mais uniquement, à concentrer ses moyens pour contrôler les niveaux dominants. Les manières de contrôle des niveaux dominants sont très diverses et vont de l'appropriation pure et simple à des formes contractuelles, plus ou moins subtiles, comme l'octroi de petits territoires exclusifs (exemple : pompe à essence) ou l'imposition de prix de vente (exemple : distribution de journaux). Un inventaire des divers moyens de contrôle vertical (autres que l'intégration) est présenté par Warren-Boulton (1979). Leur équivalence du point de vue de l'entreprise privée et du bien-être social est examinée par Blair-Kaserman (1983) selon les cas d'imperfection des marchés. Toutefois certains de leurs résultats se trouvent infirmés dès qu'on considère des ensembles d'information différents entre les agents. Le contexte informationnel (information parfaite et imparfaite) est introduit par Rey-Tirole (1986) dans leurs discussions de contraintes verticales par l'approche principal-agent. Dans leur analyse ils montrent l'importance du contexte informationnel dans le choix du contrat optimal (qui se substi- 
tuera à l'intégration). Des contraintes verticales qui étaient avant (en information symétrique) de parfaits substituts ne le sont plus. Certaines peuvent, même être non souhaitables du point de vue du monopole. D'autres peuvent avoir un effet négatif sur le bien social même quand elles sont voulues par le producteur.

À côté de ces actions offensives, le désir de contrôle de segments dominants donnera lieu à des comportements défensifs qui viseront à garder le pouvoir de domination pour soi et à entraver son passage à d'autres segments. En effet l'acteur dans un segment dominant, sur le retour, cherchera à dresser des barrières à l'entrée à son niveau et favoriser la concurrence dans les autres. Ces barrières sont qualifiées de stratégiques par opposition aux barrières légales discutées ci-dessus (section III). L'acteur en place érige des barrières car il a un avantage temporel d'entrée sur le marché. Celles-ci émanent donc des asymétries temporelles d'entrée. Elles peuvent être dressées en jouant sur des variables de capital (Tirole, 1985). Une entreprise peut accumuler avant l'entrée d'autres entreprises suffisamment de capital pour rendre cette entrée peu profitable et donc l'empêcher ${ }^{15}$. Mais pour être efficace cette accumulation de capital doit être irréversible et avoir donc valeur d'engagement. Ainsi l'entreprise déjà en place donnera l'impression d'être acculée à se battre, jusqu'au bout, en cas d'entrée. Certains contrats entre fournisseurs et clients peuvent aussi être utilisés afin de restreindre l'entrée. L'utilisation de ces contrats a été examinée de façon formelle récemment par Agion-Bolton (1985). Le contrat signé entre le fournisseur et son client stipule que si ce dernier achète à l'entrant potentiel, il devra payer un dédommagement fixé conjointement et au préalable. Ce dédommagement est un coût d'entrée supplémentaire. En effet s'il y a malgré tout entrée le nouveau vendeur devra se contenter d'un prix inférieur à celui du fournisseur déjà en place diminué du montant du dédommagement. La stratégie des filières donnera aussi lieu à des tentatives de contrôle des segments d'autres filières afin de mieux contrôler les substituts ${ }^{16}$. Cela sera réalisé soit par intégration soit en pratiquant des ventes liées.

ii) Si l'acteur n'a pas la possibilité de choisir sa localisation dans une filière il devra agir sur le déroulement du cycle de vie du produit. Il devra forcer l'évolution du cycle pour le faire entrer dans une nouvelle phase de son développement qui soit compatible avec une domination du segment où il se situe. Cette action est souvent qualifiée de rappel de l'effet de domination. En effet si la domination est localisée en amont de la filière, l'aval doit tenter d'atténuer vis-à-vis de l'utilisateur final les performances techniques du produit qui sont, rappelons-le, surtout le fait de l'amont. Il ajoutera des

15. Le terme capital doit évidemment être entendu dans un sens large ici (capital physique, clientèle, apprentissage, ...).

16. On peut citer l'exemple du producteur de matériels de bureau qui cherche à acquérir une compétence en composants électroniques lorsqu'il constate l'émergence de la bureautique. 
caractéristiques supplémentaires au produit en l'intégrant par exemple dans un ensemble de services (conseil à la clientèle, service après vente, pièces de rechange, ...). Ses efforts viseront à occulter l'existence et l'identité du producteur amont dans l'esprit des clients. Si par contre le pouvoir de domination est localisé à l'aval, l'acteur situé en amont devra le faire remonter vers lui. L'acteur amont cherchera alors à incorporer au produit d'autres performances techniques.

Ces actions se ramènent donc à une innovation technique ou commerciale qui aura pour effet de changer complètement la structure de la demande ou d'améliorer le rapport performances-prix aux yeux des demandeurs. Ce dernier type d'innovation est traité par Tirole (1985) qui affirme en outre que la motivation sera plus forte pour les entreprises concurrentielles (donc non dominantes).

L'exemple du marché de la calculatrice électronique est souvent cité pour illustrer ces stratégies de rappel de la dominance. Les fabricants amont ont réussi à faire remonter le pouvoir de domination vers eux, en remplaçant la calculatrice à quatre opérations par une nouvelle génération de calculatrices de poche à hautes performances ${ }^{17}$.

\section{c) Perspective dynamique}

Dans une perspective dynamique une stratégie de filière consistera à anticiper l'évolution du cycle de vie du produit et à déplacer constamment ses ressources avec le déplacement du pouvoir de domination. Il ne faut dès lors pas se contenter de gérer l'évolution de son segment mais innover et anticiper. Ceci implique d'identifier la future localisation de la dominance, en anticipant l'évolution du cycle de $v^{18}{ }^{18}$, s'y installer et y prendre une position forte. Mais cela implique aussi de savoir désinvestir à temps. C'est un point qui n'a pas toujours bénéficié de toute l'attention qu'il méritait. Les stratégies allemandes et japonaises se sont logiquement ordonnées dans des politiques de descente et remontée de filières. Le cas japonais est d'ailleurs très instructif à ce propos et mérite qu'on s'y arrête un moment. La « toile d'araignée » japonaise (Lorenzi-Truel, 1981) consiste à utiliser l'avance dans un secteur pour accroître techniquement, commercialement et financièrement le développement d'autres secteurs qui atteindront par la suite leur autonomie. C'est ainsi que l'explication des disparités de productivité entre le Japon et la Grande-Bretagne, par exemple, devra être trouvée dans la mise en oeuvre de leurs stratégies industrielles plutôt que dans une quelconque dotation naturelle (Brender, 1980). Au début (Boublil, 1980 ; Morvan, 1985) des années 60 , les producteurs japonais avaient fondé leur stratégie sur le développement des firmes de la filière métallique. Les profits dégagés dans l'acier, où ils occupaient la position de leader mondial, sont retenus et réinvestis dans la deuxième

17. On peut citer d'autres exemples comme l'apparition des fibres synthétiques dans la filière textile ou l'imitation de la voix humaine par les systèmes informatiques dans la filière électronique.

18. On verra ci-dessous comment des prévisions de l'évolution technologique de long terme sont possibles à partir du concept de filières. 
transformation de l'acier (automobile, construction navale). Devant les risques de surproduction dans la construction navale, ils ont pu fermer des chantiers et transférer leurs ressources et main-d'oeuvre vers l'automobile et l'électronique qui, elles, embauchaient. Cette stratégie illustre donc pour nous les vertus d'une réorientation permanente des capitaux et des hommes ${ }^{19}$.

\section{Stratégie nationale}

Si on porte, maintenant, l'approche stratégique des filières au niveau macroéconomique en considérant l'ouverture de l'économie et la mobilité des facteurs de production, elle s'avère être un moyen pour renforcer l' « autonomie nationale ${ }^{20}$. Le commerce international se réalise, dans certains cas, sur des marchés en concurrence imparfaite. En outre la hiérarchie des activités dans une filière selon des rapports de dominance-dépendance se retrouve au niveau du système productif mondial (filières transnationales). L' « autonomie nationale » nécessite dès lors que le pays ait un certain pouvoir de négociation dans ces marchés en concurrence imparfaite. Elle sera renforcée s'il a la maîtrise de quelques activités dominantes qui lui confèrent ce pouvoir de négociation. L'approche de la stratégie nationale par les filières indique les activités dominantes qu'il faut contrôler. En fonction de ces points de repère les efforts du pays viseront la spécialisation dans les activités dominantes ou celles qui préservent la cohérence de son appareil productif pour le rendre dominant.

La spécialisation internationale basée sur les seules dotations naturelles ou avantages comparatifs d'un pays est remise en question aussi bien par des économistes néo-classiques que par ceux des filières. Leurs raisons ne sont cependant pas toujours les mêmes. Du côté néo-classique on constate que la théorie des avantages comparatifs, seule, n'explique pas plusieurs observations empiriques sur le commerce international (Helpman-Krugman, 1985). Il faut dès lors faire appel à des éléments comme les économies d'échelle et la concurrence imparfaite pour $\mathrm{y}$ arriver. Ces éléments sont importants pour expliquer des phénomènes allant du volume et la composition du commerce jusqu'aux échanges intra-firmes des sociétés multi-nationales ou l'effet du commerce sur le bien-être social (Dixit-Norman, 1980 et Helpman-Krugman, 1985). Un de leurs arguments fondamentaux est que même si deux pays ont une dotation de facteurs identique, ils gagneront à se spécialiser et à s'engager dans des échanges commerciaux pour exploiter les économies d'échelle possibles. Le volume et la composition du commerce se trouvent par conséquent affectés. Cela explique alors des observations empiriques inexplicables si on ne tient compte que des dotations naturelles

19. Pour la France le travail de Vassile (1983) portant sur la stratégie de "diversification » des activités des groupes de sociétés d'un point de vue statique révèle aussi une puissante logique de filière qui leur est sous-jacente.

20. Elle invite en outre les pouvoirs publics à avoir une vision globale de l'économie qui repère les points d'étranglement (dont le retard empêche l'expansion de l'ensemble) et les noeuds de filières (qui ont un effet d'entraînement) pour éviter le saupoudrage et le gaspillage. 
et on considère des rendements constants. Les économistes de filières tout en ne niant pas ces arguments s'intéressent à un autre point. Leurs préoccupations se rapportent aux choix des activités que le pays devra implanter chez lui pour bien profiter des gains du commerce international dans un environnement de concurrence imparfaite. Pour eux il faut être attentif quant à la répartition des tâches et ne pas se contenter d'activités dominées. Il faut développer chez soi des activités prometteuses (dominantes actuellement ou dans l'avenir) même si a priori on n'a pas un avantage comparatif pour cela car dans de nombreux cas l'avantage comparatif n'est pas inné mais peut se construire ${ }^{21}$. Des arguments théoriques comme la courbe d'expérience ou des observations empiriques comme le cas japonais présenté par Brender (1980) viennent appuyer largement cette idée. Une spécialisation internationale basée sur des conceptions rigides d'avantages comparatifs risque de compromettre l'acquisition d'une compétitivité structurelle du pays et nuire à son autonomie (et l'emploi de long terme par conséquent) en le poussant vers des activités perpétuellement dominées.

Les conclusions qui découlent d'une stratégie nationale conçue en filière sont donc différentes de celles enseignées par la division internationale du travail en fonction des avantages comparatifs. Elles amènent, comme on l'a vu, les acteurs (pouvoirs publics) à choisir des segments dominants ou qui permettent à l'industrie nationale d'être dominante et non par recherche de l'optimum statique d'allocation des facteurs de production (BIPE, 1977). Ces segments permettent à l'industrie d'être dominante car ils renforcent sa cohérence. La cohérence des filières est très importante (Jacquemin-Rainelli, 1984) car des activités même fortement exportatrices, risquent d'être fragilisées par la dominance de segments étrangers en amont. Cette cohérence nationale des filières ne nécessite cependant nullement de développer toutes les filières ou d'être présent à tous les niveaux d'une filière. Elle n'implique pas l'autarcie. Elle n'est pas non plus en contradiction avec la délocalisation de certains segments vers l'étranger pour bénéficier d'avantages comparatifs pour autant que l'autonomie nationale soit préservée. Le souci est uniquement de préserver un pouvoir de négociation sur le marché. C'est d'ailleurs cette ligne de conduite qui est adoptée par les groupes industriels allemands (Montmorillon, 1980). Leur stratégie consiste à : s'implanter dans les pays industrialisés pour bénéficier de la qualité de la main-d'oeuvre et pénétrer le marché de l'intérieur, utiliser les avantages des pays en voie de développement (bas salaires, proximité des matières premières, ....) et garder en R.F.A. les activités à plus forte valeur ajoutée et qui assurent l'autonomie productive du pays. Pour guider le choix des pouvoirs publics Tollet-Kestens (1981), par exemple, proposent de classer les filières en fonction de la phase du cycle où se trouve le produit et de porter les efforts sur les segments dominants (ou qui assurent une sécurité

21. L'approche de Krugman (1984) qui analyse les interactions des politiques économiques avec le commerce international dans un monde de compétition imparfaite, rejoint dans une certaine mesure ce point de vue. 
d'approvisionnement) et pour lesquels le pays est mieux doté en facteurs de production $^{22}$.

Mais la réussite d'une politique industrielle basée sur la notion de filière dépend de l'attitude des groupes industriels et des entrepreneurs privés à son égard. Il faut donc que dans l'élaboration d'une telle politique, soit intégrée la stratégie des entreprises et les principes de leur politique. En effet il est fort possible, comme le montrent Jacquemin-Rainelli (1984), que les objectifs nationaux et ceux de l'entreprise soient contradictoires. Ces contradictions peuvent apparaître, d'après eux, si :

- la cohérence du système productif se traduit par la nécessité de contrôler l'amont (même s'il n'est pas dominant) afin de réduire le contenu en importation de l'aval, alors que la rentabilité maximale (dans le chef des entreprises) dicte un contrôle de l'aval qui comprend des activités à plus grand profit ;

- les entreprises nationales à la recherche d'une plus grande rentabilité et d'un renforcement de leur compétitivité préfèrent parfois délocaliser leurs activités, sans attention à l'autonomie nationale. Il y aura alors risque de l'accroissement de la dépendance nationale due aux flux d'importations.

Ces divergences ne sont toutefois par irréductibles. La concertation et la coordination des actions entre pouvoirs publics et entreprises au Japon par exemple ont permis la réussite de leur stratégie de filières (Brender, 1980). Les pouvoirs publics japonais tout en usant d'une planification très indicative ont aussi manipulé des instruments de politique économique tels que le contrôle de change, la politique de crédit de la Banque Centrale et la protection du marché intérieur. Ce dernier élément (protectionniste) ne fait pas l'unanimité entre les « filiéristes » (à l'image des économistes en général). Dans le cadre de l'analyse de stratégie nationale de filière, Stoffaes (1980) plaide pour une protection des « industries enfants» pour exploiter en profondeur les effets de courbes d'expérience et d'économie d'échelle. Toutefois Boublil (1980) défend que la meilleure façon de réconcilier les objectifs nationaux avec ceux de l'entreprise est de faire en sorte qu'il y ait un avantage objectif (pas de droits de douane ni de subventions) à acheter et donc à produire chez soi plutôt que de protéger des inefficacités.

\section{L'ASPECT PRÉVISIONNEL DU CONCEPT}

Nous avons vu comment les deux types de liaisons entre les segments d'une filière déterminent les actions stratégiques des différents acteurs. Mais très souvent l'action d'un acteur (privé ou public) l'engage pour l'avenir. Il doit donc être capable de prévoir

22. Mistral (1980) recommande le contrôle des industries de biens d'équipements qu'il classe à l'amont de toutes les filières. Toutefois pour soutenir cette recommandation il faut faire l'hypothèse d'une dominance permanente de l'amont. Cette hypothèse n'est pas toujours vérifiée en pratique. 
(au moins en partie) l'environnement futur et notamment l'évolution (normale) de la filière, l'impact de sa stratégie et la réaction des autres acteurs. Pour cela il doit disposer, entre autres choses, d'une base prévisionnelle propre au concept de filière. Les deux types de liaisons dans une filière et les actions stratégiques qu'ils engendrent sont utilisés comme base pour la prévision de son évolution. L'aspect prévisionnel et stratégique se révèlent par conséquent indissociables.

Les méthodes de prévision qui ont été proposées dans le cadre des filières se rapportent aussi bien au court qu'au long terme. La méthode de prévision de court terme, à situation technologique donnée, se base sur les liaisons par flux d'échanges. Les rapports dominance-dépendance et leurs conséquences stratégiques constituent quant à eux le cadre des prévisions de long terme à situation technologique variable.

\section{Prévision quantitative à court terme}

Pour la prévision quantitative on procède de façon séquentielle. Lorsqu'on a identifié et quantifié les relations d'achat-vente entre les segments d'une filière on peut mesurer l'effet, sur les échanges intermédiaires successifs, d'hypothèses sur les variations de la demande finale. Dans son principe cette méthode de prévision est donc extrêmement simple. Elle suppose, comme le modèle « inputoutput " statique, la stabilité à court terme des coefficients de ventilations des achats. Cependant même pour la prévision de court terme elle reste encore d'un intérêt limité pour plusieurs raisons. D'abord la précision des prévisions est altérée par l'existence de mouvements de stocks qui sont généralement difficiles à intégrer dans les relations économétriques ${ }^{23}$. Ensuite $^{24}$, le tableau entréessorties étant un relevé plus exaustif des relations entre secteurs que la filière, la prévision basée sur cette dernière sera nécessairement moins précise que celle issue du tableau entrées-sorties ${ }^{25}$. Enfin cette méthode de prévision, et ceci est d'ailleurs valable pour le tableau entrées-sorties, utilise implicitement l'hypothèse que chaque secteur est à même de satisfaire toute la demande qui lui est adressée. Dans le cas où les demandes intermédiaires peuvent être rationnées l'impact d'une variation de la demande finale sur le revenu agrégé risque même d'être négatif (Sprumont, 1985). De cette dernière remarque il résulte que l'application mécanique de la méthode de prévision décrite ci-dessus peut mener à de faux résultats si on ne tient pas compte des possibilités de rationnement ${ }^{26}$.

\section{Prévision technologique à long terme}

En matière de prévision technologique on distingue généralement deux approches (Malsot, 1981) :

23. Le problème reste évidemment présent aussi pour le tableau entrées-sorties.

24. Les deux raisons qui vont suivre sont plus fondamentales.

25. Cette remarque n'est évidemment plus valable lorsqu'il s'agit de prévision au niveau d'une entreprise individuelle car le tableau entrées-sorties est trop agrégé pour répondre à ses besoins.

26. C'est d'autant plus vrai que le phénomène de rationnement relève justement du court terme. 
- L'approche normative qui consiste à déterminer, par des méthodes prospectives autonomes, des performances qui doivent être satisfaites au niveau de la fonction de demande finale (voiture à faible consommation d'essence, maison à haute isolation thermique, ...). On cherche ensuite quelles innovations technologiques devraient survenir dans la filière, à l'horizon fixé, pour satisfaire les nouvelles exigences de la demande finale. Ce sont donc des normes de performances finales qui se transmettent de niveau en niveau de la filière et vont commander les solutions à mettre en oeuvre pour les satisfaire.

- L'approche exploratoire qui part d'une innovation bien précise (laser, robot, ...) et cherche quelles pourront être les applications et que seront les modifications qui en résulteront le long de la filière jusqu'à la demande finale.

Pour l'utilisation du concept de filière demande finale à des fins de prévisions technologiques de long terme, le BIPE émet deux hypothèses portant respectivement sur l'émergence d'innovations et sur le « cycle morphologique » de filières :

- Ce sont les exigences de la demande finale qui déterminent l'émergence d'innovations qui apparaîtront pour la satisfaire.

- La déformation de la morphologie de la filière dans le temps s'opère selon un cycle.

La première hypothèse conduit tout naturellement à privilégier l'approche normative qui constituera donc la méthode de prévisions technologiques dans le cadre des filières. Concernant la validité de ces hypothèses on peut dire que l'observation historique de l'émergence des innovations et de l'évolution des processus de production ne les contredit pas. En effet, la très grande majorité des innovations au cours de l'histoire des sciences et techniques est née d'une réponse à une demande existante, et le processus de croissance industrielle se caractérise historiquement par un allongement des filières. La satisfaction d'un besoin commence d'abord par être autarcique puis s'organise en une filière de plus en plus longue avec de nouveaux segments qui se spécialisent et permettent la réduction des coûts. Toutefois avec cet allongement de la filière arrive un moment où chaque nouveau segment a de plus en plus de difficultés à justifier son prélèvement sur la valeur ajoutée totale. À un stade ultime de cet allongement émerge alors une technologie nouvelle qui peut s'imposer en offrant un rapport performances/prix compétitif et en éliminant la plupart des segments existant. On assiste alors à un raccourcissement de la filière.

La prévision technologique consiste, sur base des deux hypothèses, à déterminer quelles innovations vont apparaître et si elles sont susceptibles d'allonger ou de raccourcir la filière étant donné sa situation morphologique présente et l'évolution future de la demande finale qu'elle satisfait. Nous remarquerons pour clore ce chapitre que cette méthode de prévision est indissociable des stratégies des acteurs le long de la filière qui en agissant selon leurs propres intérêts, influencent la morphologie de celle-ci. 
VI. CONCLUSION

Dans ce travail nous avons réalisé une revue générale des divers aspects du concept de filière. Tout en montrant les apports de ce concept nous avons mis en évidence un certain nombre de faiblesses voire de lacunes. De manière schématique les divers aspects du concept peuvent être classés selon trois critères :

1) l'angle d'observation qui conduit à distinguer la filière produit et la filière demande finale.

2) les méthodes d'analyse qui comprennent la méthode monographique et la méthode statistico-économétrique.

3) l'objectif de son utilisation concerne le découpage du système productif, l'analyse stratégique et la prévision quantitative et technologique.

Au terme de ce travail nous arrivons à une double constatation : richesse et lacune. Le concept de filière est à multiples facettes et offre par là une grande richesse et une véritable source d'idées pour le chercheur. Cependant toute la littérature développée autour de ce concept présente la lacune du manque de rigueur et de formalisme. La plupart des critères et d'indicateurs prêchent par excès d'empirisme qui serait loin de toute critique si parallèlement à cela un sérieux effort de formalisation rigoureuse était entrepris. Un vaste domaine reste par conséquent ouvert pour la recherche et l'analyse économique :

- Au niveau théorique : Un plus grand effort de formalisation devra être entrepris non seulement au niveau descriptif, mais aussi pour préciser certains concepts (la dominance par exemple). Pour l'analyse stratégique un pont avec le formalisme de la littérature anglo-saxonne (compétition imparfaite, contrôle vertical, ...) sera d'un très grand intérêt.

- Au niveau empirique : Les voies de recherches sont encore à peine effleurées. Celles-ci vont de tests d'hypothèses servant de base aux approches en filières à la modélisation économétrique selon une représentation en filière en passant par des méthodes de détection des filières qui évitent les critiques évoquées ci-dessus.

\section{BIBLIOGRAPHIE}

ADEFI (Association pour le développement des études sur la firme et l'industrie) (1985), L'analyse de filière, Economica, Paris, $147 \mathrm{p}$.

Agion, PH., Bolton, P. (1985), « Entry-prevention through contracts with customers », Mimeo, MIT.

ARROw, K. (1975), « Vertical integration and communication », Bell Journal of Economics, 6, pp. 173-183.

Aujac, H., (1960), « La hiérarchie des industries dans un T.E.I. et ses conséquences », Revue Économique, 2, pp. 169-238. 
BERNHARDT, I. (1977), « Vertical integration and demand variability », Journal of Industrial Economics, 25, pp. 213-229.

BIPE (Bureau d'information et de prévision économique) (1977), Une Approche de l'autonomie : Les effets de domination dans les filières industrielles, BIPE, Paris, 125 p.

Blair, D.R., Kaserman, D.L., (1983), Law and Economics of Vertical Integration and Control, Academic Press, New York, 211 p.

Boublit, A., (1980), « Filières industrielles et politique économique », Annales des Mines, 1, pp. 53-58.

BRENDER, A., (1980), « Capitalisme et organisation industrielle : quelques remarques à propos du cas japonais », Annales des Mines, 1, pp. 59-68.

CARLton, D. (1979), « Vertical integration in competitive market under uncertainty », Journal of Industrial Economics, 27, pp. 189-209.

CRETON, L. (1985), «Quelques réflexions à partir de l'analyse de la filière électronique », in ADEFI (1985), pp. 93-107.

CROCKER, K. (1983), « Vertical integration and strategic use of private information », Bell Journal of Economics, 14, pp. 236-248.

De Montmorillon, B., (1980), « Les groupes industriels allemands et la stratégie d'intégration », Annales des Mines, 1, pp. 69-76.

De Woot, Pн., (1978), « Articulation de la politique industrielle et de la stratégie des entreprises », Annales de Sciences Économiques Appliquées, 34, pp. 39-54.

Dixit, A., Norman, V. (1980), Theory of International Trade, Cambridge University Press, London, 339 p.

Gazon, J. (1976), Transmission de l'influence économique : une approche structurale, Sirey, Paris, 393 p.

HAYes, R.H., WheElwright, S.C., (1979a), « Link manufacturing process and product life cycles », Harvard Business Review, 1, pp. 133-140.

HAYES, R.H., WHEELWRIGHT, S.C., (1979b), « The dynamics of process-product life cycles », Harvard Business Review, 2, pp. 127-135.

Helpman, E., Krugman, P. (1985), Market Structure and Foreign Trade, The Harvester Press, Sussex, 271 p.

JACQUEMIN, A. (1985), Sélection et pouvoir dans la nouvelle économie industrielle, Economica, Paris, 191 p.

JACQUEMIN, A., RAINELli, M., (1984), "Filières de la nation et filières de l'entreprise », Revue Économique, 2, pp. 379-392.

Krugman, P. (1984), « The U.S. responses to foreign industrial targeting », in W. Brainard and G. Perry (éd.), Brookings Papers on Economic Activity, pp. 77-131.

LANTNER, R.J. (1974), Théorie de la dominance économique, Dunod.

Lesage, A., (1985), « Définition structurale d'une filière de production », Mondes en Développement, 47-48, pp. 215-233. 
LORENZI, J.H., TRUEL, J.L., (1981), « Se diversifier par la stratégie des filières », Harvard, L'Expansion, 13, pp. 98-107.

MALsot, J., (1980), « Filières et effet de domination dans le système productif », Annales des Mines, 1, pp. 29-40.

Malsot, J., (1981), "Stratégies de développement et filières industrielles », Société royale d'Économie Politique de Belgique, 425, pp. 1-34.

Mistral, J., (1980), « Filières et compétitivité : enjeux de la politique industrielle », Annales des Mines, 1, pp. 41-52.

Monfort, J., (1983), « À la recherche des filières de production », Économie et Statistique (INSEE), 151, pp. 3-12.

Monfort, J., Dutanly, J.C., (1983), « Les filières de production », Archives et Documents (INSEE), 67, pp. 1-193.

Morvan, Y., (1985), Fondements d'économie industrielle, Economica, Paris, $482 \mathrm{p}$.

Rey, P., Tirole, J. (1986), «Contraintes verticales : l'approche principalagent », Annales d'Économie et de Statistiques, 1, pp. 175-201.

RICHARDSON, B., (1972), « The organization of industry », Economic Journal, 82, pp. 883-896.

SouliÉ, D., (1980), « Filière de production et intégration verticale », Annales des Mines, 1, pp. 21-28.

SPRUMONT, Y., (1985), « Multiplicateur keynésien et interdépendance des secteurs de production », Annales de l'INSEE, 57, pp. 27-50.

Stoffaes, C., (1980), « Filières et stratégies industrielles », Annales des Mines, 1, pp. 9-19.

Tirole, J., (1985), Concurrence imparfaite, Economica, Paris, 136 p.

Toledano, J., (1978), « À propos des filières industrielles », Revue d'Économie Industrielle, 6, pp. 149-158.

Tollet, R., (1982), «Approches méthodologiques de la politique industrielle au travers du concept de filière », Cahiers Économiques de Bruxelles, 96, pp. 496-522.

Tollet, R., Kestens, P., (1981), « Croissance, politique industrielle et filières de production dans le cadre de la politique de développement », Communication aux Deuxièmes Journées de Technologie de l'AUPELF, 18-23 mai, 17 p.

VASSILLE, L., (1983), «Une puissante logique industrielle relie les activités des groupes », Économie et Statistiques (INSEE), 160, pp. 21-28.

W ARren-Boulton, F.R. (1978), Vertical Control of Markets, Ballinger Publishing Company, Cambridge, Massachusetts, 213 p.

Williamson, O., (1975), Markets and Hierarchies : Analysis and Antirust Implication, Free Press, New York, 286 p. 